\title{
The Accuracy and Precision of Signal Source Localization with Tetrodes
}

\author{
Chang Won Lee ${ }^{1}$, Agnieszka A. Szymanska ${ }^{2}$, Yuji Ikegaya ${ }^{3}$, and Zoran Nenadic ${ }^{4}$
}

\begin{abstract}
Four-sensor microelectrodes, commonly referred to as tetrodes, have the ability to significantly increase the signal-to-noise ratio of neuronal extracellular recordings. They also provide spatio-temporal information about extracellular action potentials (EAP) which may be used to localize and resolve individual neuronal signal sources. Since the relative position of sensors and neurons whose EAPs are recorded is not known during in vivo experiments, the accuracy and precision of neuronal source localization algorithms remain untested. In this study, electrical signals generated by a stimulator were recorded simultaneously with four recording micropipettes immersed in artificial cerebrospinal fluid. The location of the source was estimated using the multiple signal classification algorithm, with an accuracy and precision of $\sim 4 \mu \mathrm{m}$ and $\sim 7 \mu \mathrm{m}$, respectively. These results suggest that in vivo localization and resolution of individual neuronal sources is feasible.
\end{abstract}

\section{INTRODUCTION}

Multi-sensor electrodes have the ability to significantly increase the signal-to-noise ratio (SNR) of neuronal extracellular action potential (EAP) recordings. The best-known representatives of this technology are 4-sensor microelectrodes, commonly referred to as tetrodes. In comparison to their single-sensor counterparts, tetrodes have been shown to substantially improve the yield and separation of neurons in multi-unit extracellular recordings [1]. Other advantages of tetrodes include their ability to identify bursting neurons [1] and track the activity of isolated neurons over time [2]. In addition, due to their sensor geometry, tetrodes have been proposed as suitable tools for neuronal sources localization [3]. The ability to localize neurons through recorded EAPs may significantly enhance the extracellular recording process in both acute and chronic experiments. It may also lead to more accurate interpretation of scientific data.

This article presents experimental verification of the tetrode's ability to localize electrical sources in an artificial cerebrospinal fluid (aCSF). Our experimental setup utilizes four glass micropipette electrodes for recording and a single micropipette electrode for stimulation. Signals recorded by

This work was supported in part by the National Science Foundation, under Award \#1056105 to Z. Nenadic and Award \#0914303 to C.W. Lee

${ }^{1}$ C.W. Lee was with the Department of Biomedical Engineering, University of California, Irvine, CA 92697, USA. He is now with the Samsung Dallas Technology Lab, Richardson, TX 75082, USA changwonl@gmail. com

${ }^{2}$ A.A. Szymanska is with the Department of Biomedical Engineering, University of California, Irvine, CA 92697, USA a szymans @uci . edu

${ }^{3}$ Y. Ikegaya is with the Laboratory of Chemical Pharmacology, Graduate School of Pharmaceutical Sciences, University of Tokyo, Tokyo 113-0033, Japan ikegaya@mol.f.u-tokyo.ac.jp

${ }^{4} \mathrm{Z}$. Nenadic is with the Department of Biomedical Engineering and Department of Electrical Engineering and Computer Science, University of California, Irvine, CA 92697, USA znenadic@uci.edu the four micropipettes were processed using the Multiple Signal Classification (MUSIC) algorithm [4]. Estimated source locations were then compared to the true source location, as determined from a microscope image. The accuracy of these estimates $(\sim 4 \mu \mathrm{m})$ is comparable to our previously reported results [5]. In addition, since the precision of the estimated source locations $(\sim 7 \mu \mathrm{m})$ is smaller than the diameter of a typical soma, we hypothesize that this method may be able to resolve individual neuronal sources based on their EAPs.

\section{BACKGROUND AND SIGNIFICANCE}

Several neuronal source localization algorithms have been proposed and used in vivo [3], [6], [7], [8], [9]. These methods employed various approaches ranging from simple heuristic EAP generative models [6], [7], to more biophysically realistic models, such as monopole [3], dipole [9], and line source [8] approximations. However, since these experiments were performed in vivo where the exact location of neurons is unknown, the localization results could not be validated.

Source localization with a tetrode has been experimentally validated in our recent study [5]. Briefly, a commercial tetrode (Thomas Recording, Giessen, Germany) placed in close proximity to a stimulating single-sensor microelectrode, was submerged in saline and used to record a stimulus pattern. The relative positions of the tetrode and stimulator were precisely determined from microscope images by placing both electrodes in the same focal plane. However, the inability to image the location of tetrode sensors, except for the sensor at the tip, precluded direct validation of our localization algorithm. To circumvent this problem, the tetrode was moved to four different locations while its tip was held in the focal plane. The same stimulus pattern was recorded at each position, and recorded signals were aligned to the onset of the stimulus. This configuration, referred to as a "virtual tetrode," allowed data from the four different tip locations to be treated as if recorded simultaneously.

While the virtual tetrode configuration permits validation of localization results, it ignores non-stationary phenomena at the electrode-medium interface that may occur by the time the tetrode is moved from one location to another. By simultaneously recording the stimulus pattern with four spatially distributed electrodes, the present study aims to address this deficiency. It also provides a more realistic recording medium (aCSF) and a comprehensive analysis of the accuracy and precision of localization results.

The ability to localize neuronal signal sources may have significant implications to both acute and chronic extracellular recordings. In acute conditions, it may alleviate the 
tedious process of electrode positioning and adjustment. It may also improve the performance of control algorithms for autonomous neuron isolation and tracking [10], [11], [12]. In chronic conditions, tracking neuronal sources over time may allow population migration trends to be estimated. This may in turn help resolve changes in recorded firing patterns due to migration from those due to other factors such as learning and plasticity processes, reactive gliosis, and scar tissue formation.

\section{MATERIALS AND METHODS}

Five glass micropipettes (see Fig. 1) filled with aCSF were placed in a recording chamber maintained at $32^{\circ} \mathrm{C}$. Micropipettes 1-4 served for recording, while micropipette $\mathrm{S}$ served as a stimulator. The recording chamber was immersed in aCSF consisting of $127 \mathrm{mM} \mathrm{NaCl}, 26 \mathrm{mM} \mathrm{NaHCO} 3,3.3$ $\mathrm{mM} \mathrm{KCl}, 1.24 \mathrm{mM} \mathrm{KH} 2 \mathrm{PO} 4,1.0 \mathrm{mM} \mathrm{MgSO} 4,1.0 \mathrm{mM}$ $\mathrm{CaCl} 2$, and $10 \mathrm{mM}$ glucose. Electric field potentials recorded by the four micropipettes were acquired through an Axopatch 700B amplifier (Molecular Devices, Union City, CA) with a sampling rate of $20 \mathrm{kHz}$. The stimulating micropipette generated a train of ten $38-\mu \mathrm{A}$ pulses, each pulse lasting $10 \mathrm{~ms}$, followed by a 40-ms pause. To reduce the effects of noise, a total of seven such trains were recorded. Images were collected during data acquisition with an up-right microscope (Nikon, Tokyo, Japan) and captured with a cooled CCD camera (iXon DV885, Andor Technology, Belfast, UK). The experimental procedure was performed in accordance with the University of Tokyo guidelines.

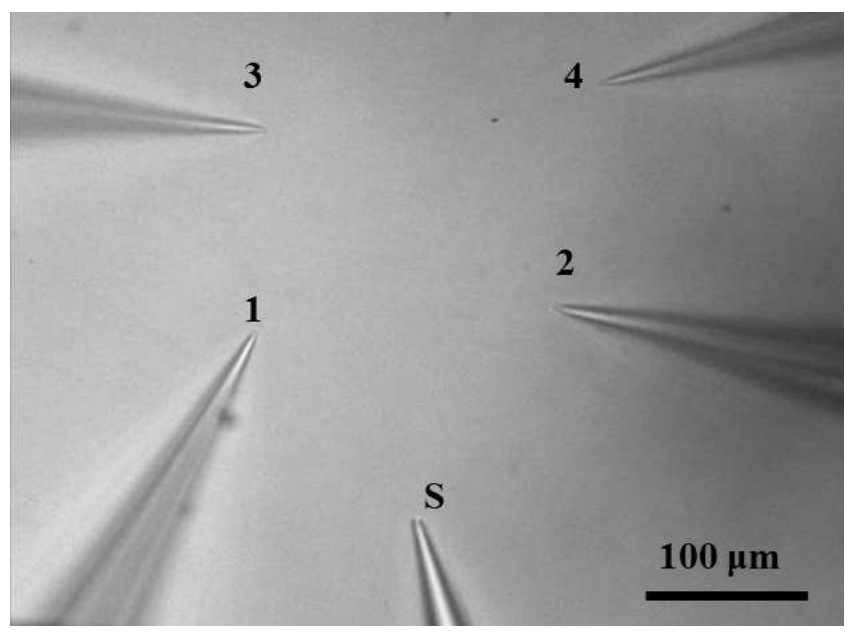

Fig. 1: Microscope image of the experimental preparation. The micropipette tips lie in the microscope focal plane. The micropipettes were subsequently brought closer by a micromanipulator (see Fig. 3).

The recorded pulse trains were aligned to the onset of the first pulse, corrected for dc offset, and averaged over the seven epochs. Source location was then estimated using the MUSIC algorithm [4], which has been successfully used in electroencephalogram [13], [14] and magnetoencephalogram [15] signal source localization. In general, the algorithm models measurements from a $c$-sensor array, $\boldsymbol{\Psi} \in$ $\mathbb{R}^{c \times 1}$, as a response of the following stochastic linear system:

$$
\boldsymbol{\Psi}(t)=\mathbf{M s}(t)+\mathbf{w}(t)
$$

where $t=1,2, \cdots, T$, ( $T$ is the number of time samples), $\mathbf{s} \in \mathbb{R}^{N_{s} \times 1}$ is a vector of signal amplitudes from $N_{s}$ sources, $\mathbf{w} \in \mathbb{R}^{c \times 1}$ is additive noise, and $\mathbf{M} \in \mathbb{R}^{c \times N_{s}}$ is a mixing matrix, whose columns, referred to as lead field vectors (LFVs), represent the system's response to a unitaryamplitude signal. Note that (1) is applicable to an arbitrary number of sensors, $c$, number of sources, $N_{s}\left(N_{s}<c\right)$, and types of sources. If the sources are dipoles, $\mathrm{s}$ consists of their dipole moment magnitudes, and if the sources are monopoles, s consists of their current amplitudes.

Assuming tetrode measurements $(c=4)$ are generated by a monopole source $\left(N_{s}=1\right)$, the LFV becomes [5], [16]:

$$
\mathbf{M}(\mathbf{r})=\frac{1}{4 \pi \sigma}\left[\frac{1}{d_{1}(\mathbf{r})} \frac{1}{d_{2}(\mathbf{r})} \frac{1}{d_{3}(\mathbf{r})} \frac{1}{d_{4}(\mathbf{r})}\right]^{\mathrm{T}}
$$

where $\sigma$ is the medium conductivity per unit length, $\mathbf{r}=$ $[x, y, z]^{\mathrm{T}}$ is the unknown source location, and $d_{i}(i=$ $1,2,3,4)$ is the distance between the source and sensor $i$. The MUSIC algorithm proceeds by finding the source location, $\mathbf{r}^{\star}$, such that the corresponding LFV is most orthogonal to the noise subspace $\mathbf{E}_{N}$ :

$$
\mathbf{r}^{\star}=\arg \min _{\mathbf{r}} \frac{\mathbf{M}^{\mathrm{T}}(\mathbf{r}) \mathbf{E}_{N} \mathbf{E}_{N}^{\mathrm{T}} \mathbf{M}(\mathbf{r})}{\mathbf{M}^{\mathrm{T}}(\mathbf{r}) \mathbf{M}(\mathbf{r})}
$$

where $\mathbf{E}_{N} \in \mathbb{R}^{4 \times 3}$ can be found by the singular value decomposition of $\boldsymbol{\Psi}_{1: T} \triangleq[\boldsymbol{\Psi}(1), \boldsymbol{\Psi}(2), \cdots, \boldsymbol{\Psi}(T)] \in \mathbb{R}^{c \times T}$. More specifically, the columns of $\mathbf{E}_{N}$ are taken as the left singular eigenvectors of $\boldsymbol{\Psi}_{1: T}$, corresponding to its smallest three singular values. While finding the minimum of the Rayleigh quotient in (3) reduces to calculating the eigenvectors of $\mathbf{E}_{N} \mathbf{E}_{N}^{\mathrm{T}}$ [17], the minimization in this case must be performed numerically due to the nonlinearity in (2). Also note that $\sigma$ cancels out in (3), and therefore the estimated source location is independent of conductivity.

The accuracy of the estimated source location was quantified by calculating the estimation error (bias) defined as:

$$
\varepsilon \triangleq\left\|E\left\{\mathbf{r}_{e}\right\}-\mathbf{r}_{t}\right\|
$$

where $\|\cdot\|$ represents the Euclidean norm, $E$ is the expectation operator (average), $\mathbf{r}_{e} \in \mathbb{R}^{3 \times 1}$ are estimated source locations, and $\mathbf{r}_{t} \in \mathbb{R}^{3 \times 1}$ is the true source location. Similarly, the precision of the estimate is quantified by a standard radius (generalized standard deviation) defined as:

$$
\delta \triangleq \sqrt{\delta_{x}^{2}+\delta_{y}^{2}+\delta_{z}^{2}}
$$

where $\delta_{x}, \delta_{y}$ and $\delta_{z}$ are the standard deviations of the $x, y$ and $z$ components of $\mathbf{r}_{e}$, respectively.

\section{RESULTS}

The recorded pulse trains, shown in Fig. 2 (Top), were processed according to the procedure described in Section III. As expected, the strongest signals were recorded by sensors 1 and 2, which lied closest to the source [see Fig. 3 (Left)]. 

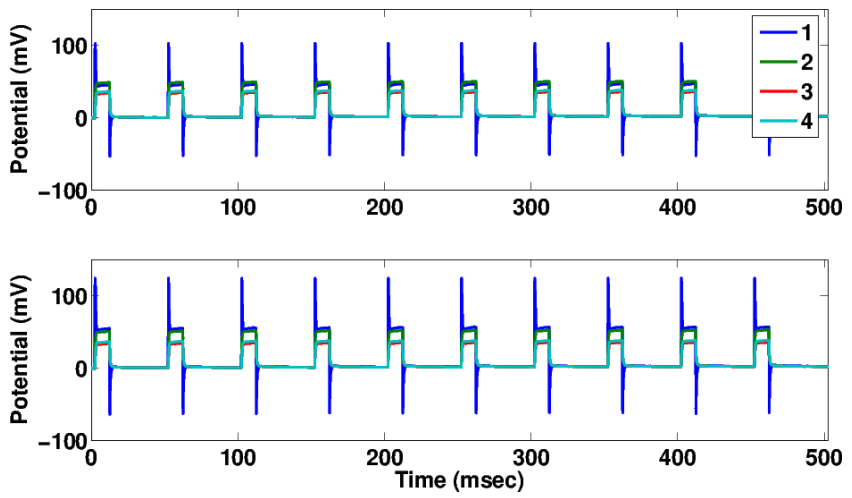

Fig. 2: (Top) A train of 10 pulses recorded by the four micropipettes (1-4). The traces represent averages over seven epochs. (Bottom) The same signals after ICF adjustment.

The 10-msec data, corresponding to each of the 10 pulses, were then used as input to the MUSIC algorithm, yielding one estimated source location for each pulse [Fig. 3 (Center)]. The average over the 10 estimated sources was (-6.45, $43.02,4.70) \mu \mathrm{m}$ [B in Fig. 3 (Left)], with the origin defined at the source, S. This corresponds to an error, $\varepsilon$, of 43.75 $\mu \mathrm{m}$. While Fig. 3 (Center) shows that the solutions were consistent in the x-y plane ( $\left.\delta_{x}=0.03 \mu \mathrm{m}, \delta_{y}=0.15 \mu \mathrm{m}\right)$, the variance along the $\mathrm{z}$-axis was high $\left(\delta_{z}=22.25, \mu \mathrm{m}\right)$, yielding an overall standard radius, $\delta$, of $22.25 \mu \mathrm{m}$.

This relatively large bias $(\sim 44 \mu \mathrm{m})$ is consistent with our previous study (bias: $\sim 41 \mu \mathrm{m}$ ) [5]. It indicates that the modeling assumptions and, in turn, the constraint imposed on the LFV (2) may not hold. In the simplest scenario, this bias can be attributed to medium inhomogeneity and mitigated by allowing each source-sensor path to have a different conductivity value. This redefines the LFV (2) as:

$$
\overline{\mathbf{M}}(\mathbf{r})=\frac{1}{4 \pi \sigma}\left[\frac{1}{k_{1} d_{1}(\mathbf{r})} \frac{1}{k_{2} d_{2}(\mathbf{r})} \frac{1}{k_{3} d_{3}(\mathbf{r})} \frac{1}{k_{4} d_{4}(\mathbf{r})}\right]^{\mathrm{T}}
$$

where $k_{i}(i=1,2,3,4)$ is a constant making each conductivity value a multiple of some baseline conductivity $\sigma$. This multiplier, referred to as an inhomogeneity correction factor (ICF), can be found experimentally, provided the distance, $d_{i}(i=1,2,3,4)$, is known. By assuming that one of the stimulator-sensor paths has the baseline conductivity, $k_{4}=1$, the remaining ICFs can be found using (1) and (6), and taking the expectation of $\Psi$ over the noise distribution:

$$
k_{i}(t)=\frac{d_{4} E\left\{\Psi_{4}(t)\right\}}{d_{i} E\left\{\Psi_{i}(t)\right\}}, \quad i=1,2,3
$$

where $\Psi_{i} \in \mathbb{R}$ is the $i$ th component of $\boldsymbol{\Psi}$. Although $k_{i}$ depends on time, we have shown that its values remain fairly stable over time [5] and can be estimated by taking the median value of $k_{i}(t)$. This yielded $k_{1}=1.20, k_{2}=1.04$ and $k_{3}=0.99$.

The forward model (1) is then modified as:

$$
\overline{\mathbf{\Psi}}(t)=\mathbf{M s}(t)+\overline{\mathbf{w}}(t)
$$

where the components of $\overline{\boldsymbol{\Psi}}$ are $\bar{\Psi}_{i}=k_{i} \Psi_{i}$, and those of $\overline{\mathbf{w}}$ are $\bar{w}_{i}=k_{i} w_{i}$. The MUSIC algorithm can then be executed using ICF corrected signals, $\overline{\mathbf{\Psi}}$. Fig. 2 shows a comparison of signals before and after ICF adjustment.

The localization results significantly improved upon ICF correction. The average estimated source location was $(0.20$, $0.61,3.78) \mu \mathrm{m}$ [A in Fig. 3 (Left)], with corresponding error $\varepsilon=3.83 \mu \mathrm{m}$. This is also highly consistent with our previously reported results (error: $\sim 3 \mu \mathrm{m}$ ) [5]. Likewise, ICF adjustment substantially reduced localization variance ( $\delta_{x}=0.40 \mu \mathrm{m}, \delta_{y}=3.50 \mu \mathrm{m}$, and $\delta_{z}=6.27 \mu \mathrm{m}$ ), yielding a standard radius, $\delta$, of $7.20 \mu \mathrm{m}$. The clustering of these sources is shown in Fig. 3 (Right).

\section{DISCUSSION}

The sole purpose of ICF adjustment in our analysis was to validate the performance of the localization algorithm. Since determining ICFs relies on known distances between the sensors and the source, ICF adjustment cannot be performed on blind data collected in vitro or in vivo. Source localization based on unadjusted signals, however, is likely to result in a relatively large bias and increased variance. We hypothesize that increased localization accuracy and precision could be accomplished by prewhitening or interference suppression prior to source localization [13].

As suggested in Section IV, the bias is believed to have been primarily caused by medium inhomogeneity, although other factors such as medium anisotropy or non-uniform sensor impedances could play a role. However, preliminary evidence suggests that impedance inconsistency is not likely to be the cause, as similar bias values have been observed in our previous study [5], performed using a single sensor placed at four recording locations.

Another potential source of bias could be a failure of the monopole model to accurately describe the source. Our preliminary in silico work [18], as well as in vivo studies performed by Mechler and Victor [19], indicate that discrepancies between monopole-generated signals and measured neuronal signals often result in localization solutions biased toward the sensors. However, the presence of localization bias is not specific to neuronal sources, as it was also observed in our previous study [5], with the stimulator designed to mimic a monopole source. Likewise, the distant placement of the stimulator's reference electrode in the present study suggests that a monopole should be a good approximation of the electrical field potential in the vicinity of the stimulator. Based on the above observations, we conclude that the monopole model is not the source of bias in this and other studies.

Finally, the main cause for the relatively low precision of source localization prior to ICF adjustments appears to lie in the planar sensor geometry. Our unpublished work [18], simulating 2D sensor arrangement in silico, indicated that planar arrays generally lack sufficient information about the source to resolve the third dimension. Depending on the exact setup geometry and SNR, the sources often tend to cluster at two geometrically mirrored locations on either side of the sensor plane. This was observed in the present study, with the $\mathrm{z}$-coordinates across 10 localization trials clustered at 

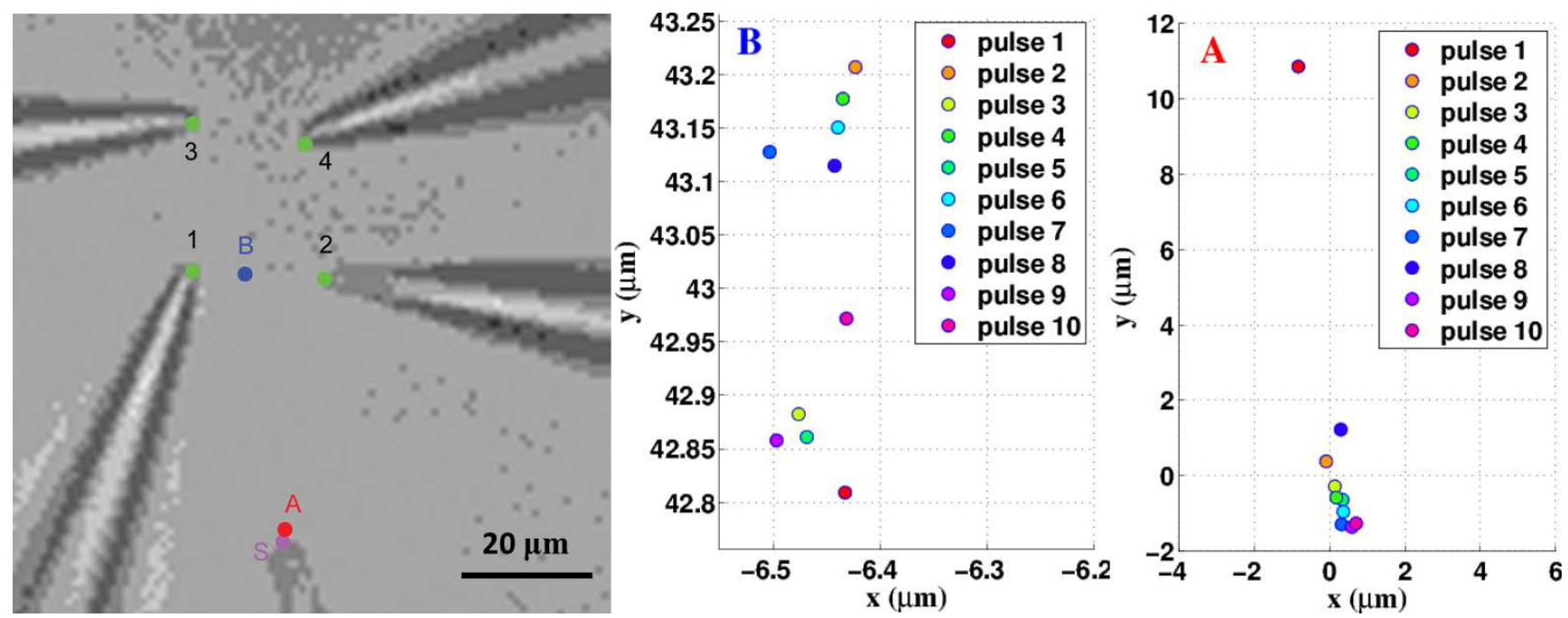

Fig. 3: (Left) The final sensor-stimulator configuration. The sensors (the tips of micropipettes 1-4) are marked in green, and the source (the tip of the stimulating micropipette) is marked in magenta. The average of estimated sources is marked by B. The equivalent point after ICF adjustment is marked by A. (Center) The estimated locations of 10 sources corresponding to the 10 pulses before ICF adjustment. (Right) The equivalent plot for the pulse train after ICF adjustment.

either $\sim 23 \mu \mathrm{m}$ or $-23 \mu \mathrm{m}$. Note that this was the main reason for the relatively large standard radius seen prior to ICF adjustment (Section IV). This deficiency can be easily mitigated by using a 3D sensor array.

\section{CONCLUSION}

The work presented here builds on previously published MUSIC localization studies, and further reaffirms the MUSIC algorithm's ability to accurately localize single electrical sources. Future studies will concentrate on expanding the algorithm's ability to characterize the intensity of the source, and applying it to in vitro and in vivo recording experiments.

\section{REFERENCES}

[1] C.M. Gray, P.E. Maldonado, M. Wilson, and B. McNaughton. Tetrodes markedly improve the reliability and yield of multiple single-unit isolation from multi-unit recordings in cat striate cortex. J. Neurosci. Meth., 63(1-2):43-54, 1995.

[2] A.A. Emondi, S.P. Rebrik, A.V. Kurgansky, and K.D. Miller. Tracking neurons recorded from tetrodes across time. J. Neurosci. Meth., 135(12):95-105, 2004.

[3] M.I. Chelaru and M.S. Jog. Spike source localization with tetrodes. J. Neurosci. Meth., 142(2):305-315, 2005.

[4] R. Schmidt. Multiple emitter location and signal parameter estimation. IEEE Trans. Antennas Propagat., 34(3):276-280, 1986.

[5] C.W. Lee, C.E. King, S.C. Wu, A.L. Swindlehurst, and Z. Nenadic. Signal source localization with tetrodes: Experimental verification. In Proc. of the 33rd Annual International Conference of the IEEE Engineering in Medicine and Biology Society, pages 67-70, 2011.

[6] J. Csicsvari, D.A. Henze, B. Jamieson, K.D. Harris, A. Sirota, P. Bartho, K.D. Wise, and G. Buzsaki. Massively parallel recording of unit and local field potentials with silicon-based electrodes. $J$. Neurophysiol., 90(2):1314-1323, 2003.
[7] P. Bartho, H. Hirase, L. Monconduit, M. Zugaro, K.D. Harris, and G. Buzsaki. Characterization of neocortical principal cells and interneurons by network interactions and extracellular features. $J$. Neurophysiol., 92(1):600-608, 2004.

[8] Z. Somogyvari, L. Zalanyi, I. Ulbert, and P. Erdi. Model-based source localization of extracellular action potentials. J. Neurosci. Meth., 147(2):126-137, 2005.

[9] F. Mechler, J.D. Victor, I. Ohiorhenuan, A.M. Schmid, and Q. Hu. Three-dimensional localization of neurons in cortical tetrode recordings. J. Neurophysiol., 106(2):828-848, 2011.

[10] Z. Nenadic and J. W. Burdick. A control algorithm for autonomous optimization of extracellular recordings. IEEE Trans. Biomed. Eng., 53(5):941-955, 2006

[11] J.G. Cham, E.A. Branchaud, Z. Nenadic, B. Greger, R.A. Andersen, and J.W. Burdick. Semi-chronic motorized microdrive and control algorithm for autonomously isolating and maintaining optimal extracellular action potentials. J. Neurophysiol., 93(1):570-579, 2005.

[12] J.W. Burdick, J.G. Cham, Z. Nenadic, E.A. Branchaud, M.T. Wolf, and R.A. Andersen. Prosthetic devices and methods and systems related thereto, 2012. US Patent 8,095,210.

[13] S.C. Wu, A.L. Swindlehurst, P.T. Wang, and Z. Nenadic. Projection versus prewhitening for EEG interference suppression. IEEE Trans. Biomed. Eng., 59(5):1329-1338, 2012.

[14] S.C. Wu, A.L. Swindlehurst, P.T. Wang, and Z. Nenadic. Efficient dipole parameter estimation in EEG systems with near-ML performance. IEEE Trans. Biomed. Eng., 59(5):1339-1348, 2012.

[15] J.C. Mosher and R.M. Leahy. Recursive MUSIC: A framework for EEG and MEG source localization. IEEE Trans. Biomed. Eng., 45(11):1342-1354, 1998

[16] C.W. Lee, H. Dang, and Z. Nenadic. An efficient algorithm for current source localization with tetrodes. In Proc. of the 29th Annual International Conference of the IEEE Engineering in Medicine and Biology Society, pages 1282-1285, 2007.

[17] R.A. Horn and C.A. Johnson. Matrix Analysis. Cambridge University Press, New York, 1985.

[18] Chang W. Lee. A method for neural source identification. PhD thesis, University of California, Irvine, 2012.

[19] F. Mechler and J.D. Victor. Dipole characterization of single neurons from their extracellular action potentials. J. Computat. Neurosci. 32(1):73-100, 2012. 